Research Article

\title{
Effectiveness and Safety of Botulinum Toxin Type A in the Treatment of Androgenetic Alopecia
}

\author{
Yaguang Zhou, Shui Yu, Jimin Zhao, Xinyue Feng, Meinan Zhang, and Zigang Zhao \\ Department of Dermatology, Hainan Hospital of PLA General Hospital, Sanya, Hainan Province, China \\ Correspondence should be addressed to Zigang Zhao; zhaozigang301@126.com
}

Received 18 April 2020; Revised 19 June 2020; Accepted 10 July 2020; Published 4 August 2020

Academic Editor: Pradeep Tyagi

Copyright (C) 2020 Yaguang Zhou et al. This is an open access article distributed under the Creative Commons Attribution License, which permits unrestricted use, distribution, and reproduction in any medium, provided the original work is properly cited.

\begin{abstract}
Background. Androgenetic alopecia (AGA) represents the most frequent clinical complaint encountered by dermatologists and is characterized by a progressive miniaturization of the hair follicle. However, the efficacy and safety of current medical treatment remain limited, and more personalized therapeutic approaches for AGA are needed. Therefore, the present study is aimed at investigating the efficacy and safety of botulinum toxin type A (BTA) in patients with AGA. Methods. 63 patients with AGA meeting the inclusion criteria were included in this study and treated with BTA injection or BTA injection combined with oral finasteride (FNS). In the scalp, 30 sites were injected with $100 \mathrm{U}$ of BTA in each site and patients received BTA after every 3 months for a total of 4 times. Hair counts, head photographs, evaluation scores, and self-assessment were assessed in patients with AGA. Results. Hair counts in both groups at all time points were significantly higher as compared with those before treatment. After 4 times of treatment, hair counts in the BTA+FNS group were higher than those in the BTA group. Hair growth and density were significantly augmented, and the area of hair loss was attenuated after each treatment as revealed by head photographs. The effective rates of BTA and BTA+FNS groups were $73.3 \%$ and $84.8 \%$, respectively, following 4 times treatment. Conclusion. BTA is a safe and effective therapeutic strategy for the treatment of AGA without adverse effects, and BTA combined with FNS exhibited a superior therapeutic effect than BTA alone.
\end{abstract}

\section{Introduction}

Hair loss represents the most frequent and distressing clinical complaint encountered by dermatologists in clinical practice. Androgenetic alopecia (AGA), telogen effluvium, and alopecia areata are the three most common types of hair loss, with AGA being the most prevalent type in dermatology practice. AGA or pattern baldness, characterized by a progressive miniaturization of the hair follicle, is a nonscarring hair loss disorder that predominantly affects up to $80 \%$ of men and $50 \%$ of women during adolescence and postadolescence [1-3]. The prevalence varies across ethnic groups, with higher prevalence rates reported in Caucasian men (50\%) and women (19\%) than in Asian and black men. The pathogenesis of AGA is highly variable and remains elusive; however, the presence of dihydrotestosterone (DHT) and degree of genetic predisposition plays a causative role in its development $[4,5]$. There are several traditional treatment options available for the treatment of AGA; however, their effectiveness remains limited.
Therefore, a safe and effective treatment modality with fewer side effects that can significantly benefit patients with AGA in a dermatology practice setting is highly desirable.

Accumulating studies have indicated that increased levels of DHT and overexpression of the androgen receptor (AR) gene were found to be associated with AGA [6]. Furthermore, $5 \alpha$-reductase present within the dermal papilla plays a crucial role in the intrafollicular conversion of systemic and local testosterone into DHT. DHT binds to the follicular AR, which eventually induces signaling pathways associated with progressive miniaturization and hair loss [7]. Thus, attenuation of levels of DHT is vital for the prevention and treatment of AGA.

Botulinum toxin type A (BTA) is a highly potent neurotoxin that selectively prevents the release of acetylcholine at the neuromuscular junction and is most extensively used in the treatment of a various dermatological disorder $[8,9]$. A recent, alternative approach is the injection of BTA into the scalps of patients with AGA. Though a paucity of studies 
showed that BTA has been tried for AGA management, they had limitations including small sample sizes, unclear evaluation standards, the loss to follow-up of many patients, and lack of combination therapy with other drugs. Therefore, more studies are required to establish the role of BTA in the treatment of AGA.

The present study is aimed at evaluating the safety and effectiveness of BTA on the treatment of AGA.

\section{Methods}

2.1. Participants. This study enrolled 63 patients who were presented and diagnosed with AGA at the Department of Dermatology, Hainan Hospital of People's Liberation Army General Hospital, between February 2017 and February 2019. Patients with AGA were randomly divided into BTA and BTA+finasteride (FNS) groups (We have used a simple randomization grouping method, which was to directly randomize the subjects, and generate random numbers by computer for randomization, without any restrictions or interventions or adjustments in advance or in the implementation process). AGA patients in the BTA group $(n=30)$ received BTA (Botox; Allergan, USA) injection, and AGA patients in the $\mathrm{BTA}+\mathrm{FNS}$ group $(n=33)$ received $\mathrm{BTA}$ injection in combination with oral FNS (Propecia; MSD, $\mathrm{UK} ; 1 \mathrm{mg} / \mathrm{d})$. Inclusion criteria were as follows: men aged 18-60 years with a confirmed diagnosis of AGA; AGA diagnosis was evaluated following Norwood Hamilton grade II-IV criteria, patients with no history of treatment with drugs that are known to interfere with BTA within last 6 months; and willingness to provide pictures and follow-up studies. Patients were excluded if presented with severe diseases of internal organs, eyes, or skin; neuromuscular system diseases; inflammation, infection, or unhealed wounds on the skin around the site of injection on the head; systematic treatment with corticosteroids or other immunosuppressants and immunomodulators in the past 3 months; and phobic about treatment with BTA.

2.2. Study Design. The study was approved by the Ethics Committee of Hainan Hospital of People's Liberation Army General Hospital. This study conformed to the Declaration of Helsinki, and all participants provided written informed consent. All patients received the treatment for 12 months and were followed up every 3 months, 4 times in total during and after completion of the treatment. Any alteration in hair growth of the head and associated adverse events were recorded during the treatment and at each follow-up visit. Representative photographs of the head were acquired before and after each treatment.

2.3. Botulinum Toxin Injection. BTA $(100 \mathrm{U} / \mathrm{ml}$, Botox; Allergan, USA) was diluted with $3 \mathrm{ml}$ of $0.9 \%$ normal saline. The scalp was sterilized with iodophor, and 30 injection target sites (located in the frontal muscle, temporal muscle, periauricular muscle, and occipital muscle) were marked with gentian violet, and each injection site was $1.5-2 \mathrm{~cm}$ apart. BTA at the total injection dose of $100 \mathrm{U} / \mathrm{ml}$ was administered intramuscularly using an insulin syringe of $40 \mathrm{U} / \mathrm{ml}$ capaci- ties. Participants received BTA injection every 3 months, 4 times in total.

2.4. Efficacy Assessment. The efficacy of treatment was evaluated using the hair counts statistics and objective evaluation score of the photographs of the head before and after treatment by dermatologists as the primary outcome and the self-evaluation score of the patients as the secondary outcome.

2.4.1. Hair Counts Statistics. Professional photographic camera (Canon EOS 90d) equipped with a lens (Canon ef-s18$135 \mathrm{~mm} \mathrm{f/3.5-5.6} \mathrm{is} \mathrm{USM)} \mathrm{and} \mathrm{tripod} \mathrm{(to} \mathrm{ensure} \mathrm{the} \mathrm{identical}$ angle and distance for image acquisition) was used for the study. After each treatment, the hairstyle of the patients was kept consistent with that before the treatment. The hair counts in the test before each session and by the end of follow-up were determined according to Canfield's statistical method by an experienced dermatologist [10]. The measurement area of the scalp was identified by the minitattoo, and hair count assessments were performed using a $2 \mathrm{~cm}^{2}$ circular template centered over the minitattoo.

2.4.2. Evaluation Score of Head Photos. To assess the improvement in hair growth, two independent dermatologists evaluated the photographic images of the head before each session and by the end of follow-up. Based on the change in hair density in the photographs of the head, the effects of treatment were evaluated using the 4-point scale with 0 defined as poor, 1 as fair, 2 as good, and 3 as excellent. The effective rate was calculated by (good + excellent $) /($ poor + fair + good + excellent) $\times 100 \%$.

2.4.3. Self-Assessment Evaluation of Patients. Before and after each treatment, patients were requested to complete a questionnaire. Data on hair loss, dandruff, and scalp oil secretion were also recorded.

2.5. Evaluation of Adverse Effects. Data on localized adverse effects including inflammation, erythema, edema, and blisters at the injection site and systemic adverse effects including fever, headache, chest tightness, and nausea were collected.

2.6. Statistical Analysis. Quantitative data were expressed as mean \pm standard deviation and compared using Student's $t$ -test or repeated measures analysis of variance (ANOVA). Qualitative data were expressed as frequency (percentage) and compared using the chi-square test. All data were analyzed using SPSS 17.0. A $P$ value of $<0.05$ was considered statistically significant.

\section{Results}

A total of 63 patients with AGA enrolled to this study. Patients' demographic and clinical characteristics of both groups were summarized in Table 1 . There was no significant difference in age, BMI, age hair loss first noticed, and disease durations between BTA and BTA+FNS groups. The Norwood-Hamilton grade and hair counts before treatment also showed no difference between the two groups. 
TABle 1: Demographic and clinical characteristics.

\begin{tabular}{|c|c|c|c|}
\hline & BTA $(n=30)$ & $\mathrm{BTA}+\mathrm{FNS}(n=33)$ & $P$ value \\
\hline Age (years) & $38.47 \pm 10.13$ & $40.06 \pm 11.85$ & 0.571 \\
\hline BMI $\left(\mathrm{kg} / \mathrm{m}^{2}\right)$ & $23.12 \pm 4.54$ & $23.89 \pm 3.72$ & 0.463 \\
\hline Age hair loss first noticed (years) & $31.27 \pm 6.35$ & $29.67 \pm 8.01$ & 0.386 \\
\hline Disease duration (years) & $8.85 \pm 7.17$ & $7.83 \pm 7.90$ & 0.596 \\
\hline \multicolumn{4}{|c|}{ Norwood-Hamilton grade, $n(\%)$} \\
\hline II vertex & $9(30.0)$ & $7(21.2)$ & \\
\hline III & $13(43.3)$ & $15(45.4)$ & \\
\hline IV & $8(26.7)$ & $11(33.3)$ & 0.696 \\
\hline Hair counts before treatment $\left(\mathrm{root} / \mathrm{cm}^{2}\right)$ & $180.57 \pm 26.53$ & $178.21 \pm 24.33$ & 0.715 \\
\hline
\end{tabular}

BTA: botulinum toxin type A; BTA+FNS: botulinum toxin type A+finasteride.

3.1. Efficacy. Hair counts in both groups at all time points were significantly higher compared with those before treatment (all $P<0.05$ ) and further increased gradually with prolongation of the treatment time (Table 2). After an overall treatment for 4 times, hair counts in the BTA+FNS group were $234.01 \pm 27.35 \mathrm{root} / \mathrm{cm}^{2}$ and $218.26 \pm 30.59 \mathrm{root} / \mathrm{cm}^{2}$ in the BTA group, and the hair counts of both groups were significantly different $(P<0.05)$. However, no significant difference was observed in hair counts between two groups when measured after treatment for once, twice, and three times. Representative photographic images of patients with AGA before and after each treatment were presented in Figure 1 . Hair density was significantly augmented, and the target area of hair loss was attenuated after each treatment, suggesting distinctive improvement with BTA and BTA + FNS treatment. The effective rates for BTA and BTA+FNS groups were $73.3 \%$ and $84.8 \%$ after 4 times treatment, respectively; however, no significant difference in the efficacy of BTA and BTA+FNS on patients with AGA was observed between the two groups (Table 3 ).

3.2. Patient Satisfaction. After treatment, 16 AGA patients in BTA group and 23 patients in BTA+FNS group reported that they exhibited moderate and marked improvement in fewer symptoms of scalp oil secretion, pruritus, and dandruff (Figure 2(a)). Moreover, 23 AGA patients in BTA group and 27 AGA patients in BTA+FNS group experienced a moderate and marked reduction in hair loss as compared with before treatment (Figure 2(b)).

3.3. Safety and Tolerability. There were no severe adverse events occurred in all patients. One patient in the BTA group developed headache, possibly related to study medication. Injection site events (pain, erythema, or edema) were developed in 2 patients and 1 patient in the BTA and BTA +FNS group, respectively. A slight breathlessness and nausea associated with BTA injection was reported by 1 patient in $\mathrm{BTA}+\mathrm{FNS}$ group.

\section{Discussion}

AGA is an androgen-dependent and predominantly genetically determined trait characterized by a chronic and pro- gressive miniaturization of hair follicles [11]. Previous epidemiological studies indicated that men with paternal AGA history is an important risk factor for early onset of AGA in men, while men with a maternal family history were associated with poor prognosis $[12,13]$. The whole-genome sequencing and mapping studies have identified several susceptibility loci for AGA; however, the pathogenic genes associated with AGA have not been identified so far [14]. Although the etiology and pathogenesis of AGA remain elusive, recent studies demonstrated that androgen functions as a crucial factor in the pathogenesis of AGA [15]. An increasing number of studies have suggested that 5-alpha reductase converts testosterone to DHT, which binds to hair follicular ARs and leads to the activation of genes that induces miniaturization of the hair follicle and disappearance of hair follicle atrophy, alopecia, and ultimately baldness [16, 17]. Furthermore, the symptoms of AGA could be aggravated by factors such as insufficient blood supply to the scalp, infection in hair follicles, psychological stress or disorder, irregular sleep, and insomnia.

At present, the treatment of AGA mainly includes systemic drugs (such as FNS and spironolactone), external drugs (such as minoxidil), and other alternative methods (hair transplantation, platelet-rich plasma, and low-energy laser) [18]. Notably, combination therapy has been proposed to achieve the best efficacy expediently. However, oral medication requires long-term use and exhibit certain associated side effects, topical medication has limited and inaccurate efficacy, and invasive treatment carries surgical risks [19-21]. And hair loss is associated with considerable psychological and emotional distress and decreased quality of life. Therefore, a safe and effective treatment modality with fewer side effects that can greatly benefit patients with AGA in a dermatology practice setting is highly desirable.

Botulinum toxin is one of the highly effective neurotoxins produced by the bacterium Clostridium botulinum. BTA prevents the release of acetylcholine and many other neurotransmitters at the presynaptic neuromuscular junction [22]. BTA has been extensively used in the dermatology clinic for wrinkle reduction, facial muscle adjustment, hyperhidrosis, correction of masseter hypertrophy, and gastrocnemius hypertrophy. Besides, long-term effects of muscles surrounding the affected scalp (including the frontal, occipital, 
TABLE 2: Evaluation of hair counts between two groups at different time.

\begin{tabular}{|c|c|c|c|}
\hline & BTA $(n=30)$ & $\mathrm{BTA}+\mathrm{FNS}(n=33)$ & $P$ value \\
\hline \multicolumn{4}{|c|}{ Hair counts at different time $\left(\mathrm{root} / \mathrm{cm}^{2}\right)$} \\
\hline 0 (before treatment) & $180.57 \pm 26.53$ & $178.21 \pm 24.33$ & 0.715 \\
\hline 3 months (once treatment) & $196.03 \pm 32.89$ & $205.78 \pm 28.50$ & 0.212 \\
\hline 6 months (twice treatment) & $208.04 \pm 27.00$ & $220.44 \pm 27.06$ & 0.074 \\
\hline 9 months (three times treatment) & $214.83 \pm 31.17$ & $228.31 \pm 30.99$ & 0.091 \\
\hline 12 months (four times treatment) & $218.26 \pm 30.59$ & $234.01 \pm 27.35$ & 0.035 \\
\hline${ }^{\mathrm{a} P}$ value & 0.015 & $<0.001$ & \\
\hline${ }^{\mathrm{b}} P$ value & $<0.001$ & $<0.001$ & \\
\hline${ }^{\mathrm{c}} P$ value & $<0.001$ & $<0.001$ & \\
\hline${ }^{\mathrm{d}} P$ value & $<0.001$ & $<0.001$ & \\
\hline
\end{tabular}

BTA: botulinum toxin type A; BTA+FNS: botulinum toxin type A+finasteride; ${ }^{\mathrm{a}} P$ value was derived from comparisons between 0 and 3 months; ${ }^{\mathrm{b}} P$ value was derived from comparisons between 0 and 6 months; ${ }^{\mathrm{c}} P$ value was derived from comparisons between 0 and 9 months; ${ }^{\mathrm{d}} P$ value was derived from comparisons between 0 and 12 months.
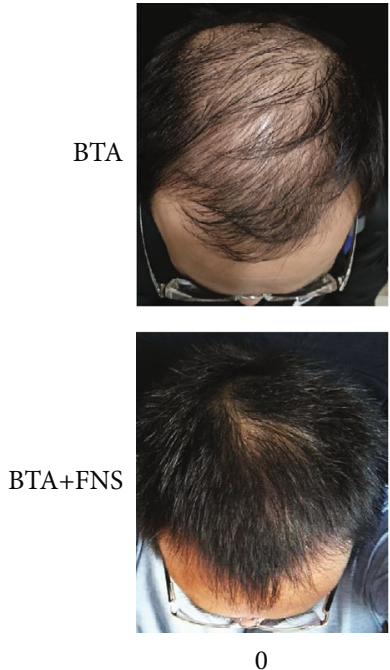

0
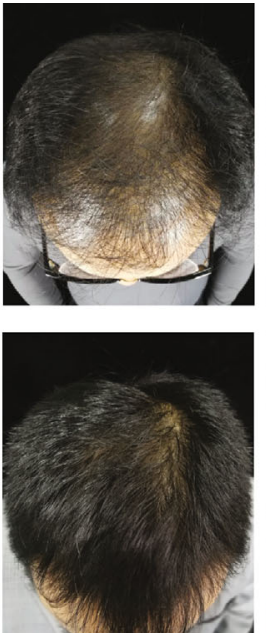

3 months
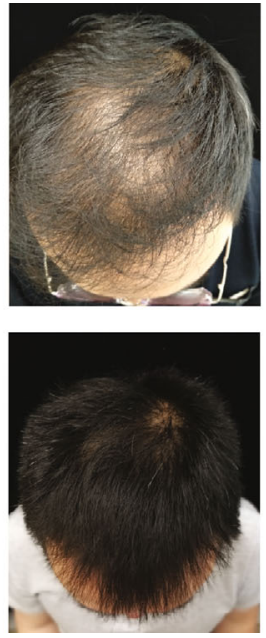

6 months
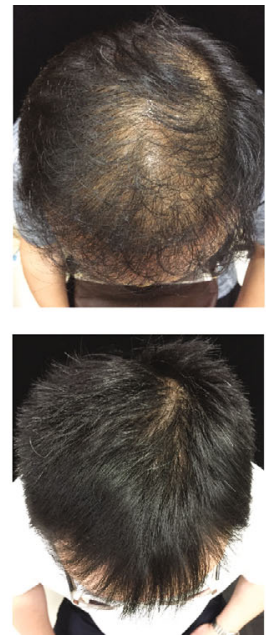

9 months
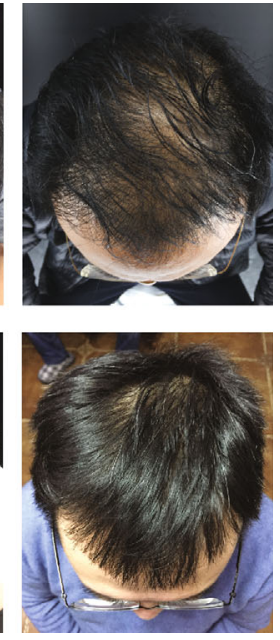

12 months

Figure 1: Clinical effectiveness between two groups at different time. BTA: botulinum toxin type A; BTA+FNS: botulinum toxin type A + finasteride.

TABLE 3: Evaluation score and effective rate between two groups.

\begin{tabular}{cccc}
\hline & BTA $(n=30)$ & BTA+FNS $(n=33)$ & $P$ value \\
\hline \multicolumn{4}{c}{ Evaluation score after 4 times treatment, $n(\%)$} \\
0 & $3(10.0)$ & $2(6.1)$ & \\
1 & $5(16.7)$ & $3(9.1)$ & \\
2 & $9(30.0)$ & $10(30.3)$ & \\
3 & $13(43.3)$ & $18(54.5)$ & 0.259 \\
\hline
\end{tabular}

BTA: botulinum toxin type A; BTA+FNS: botulinum toxin type A + finasteride.

periauricular, and temporal muscles) tighten the affected scalp, resulting in reduced blood flow to the terminal vessels at the top of the head and forehead, which eventually leads to a hypoxic state in these affected areas [23]. Moreover, DHT is prone to activation under hypoxic conditions, and it is the most crucial factor that terminates hair follicles and causes hair loss. Previous studies suggested that there were hemodynamic abnormalities including microvascular dysfunction and reduced blood flow in the alopecia region of AGA patients [24]. Therefore, improving the scalp blood supply may stimulate hair growth. Possibly, BTA may relax the muscles around the head, increase blood flow and oxygen concentration in the alopecia area, and further inhibit the activation of DHT, ultimately leading to a reduced occurrence of hair loss. Moreover, a high concentration of oxygen can stimulate the hair follicle into the growth phase, resulting in hair regeneration [25-27]. In the present study, we demonstrated that the treatment of the alopecia region with BTA significantly increased hair counts of patients with AGA, and the effect of BTA alone was lower compared to that of BTA combined with FNS. Hair growth and density were also significantly elevated after each treatment as revealed from representative photographs of patients with AGA, and both groups presented a high effective rate. Our 


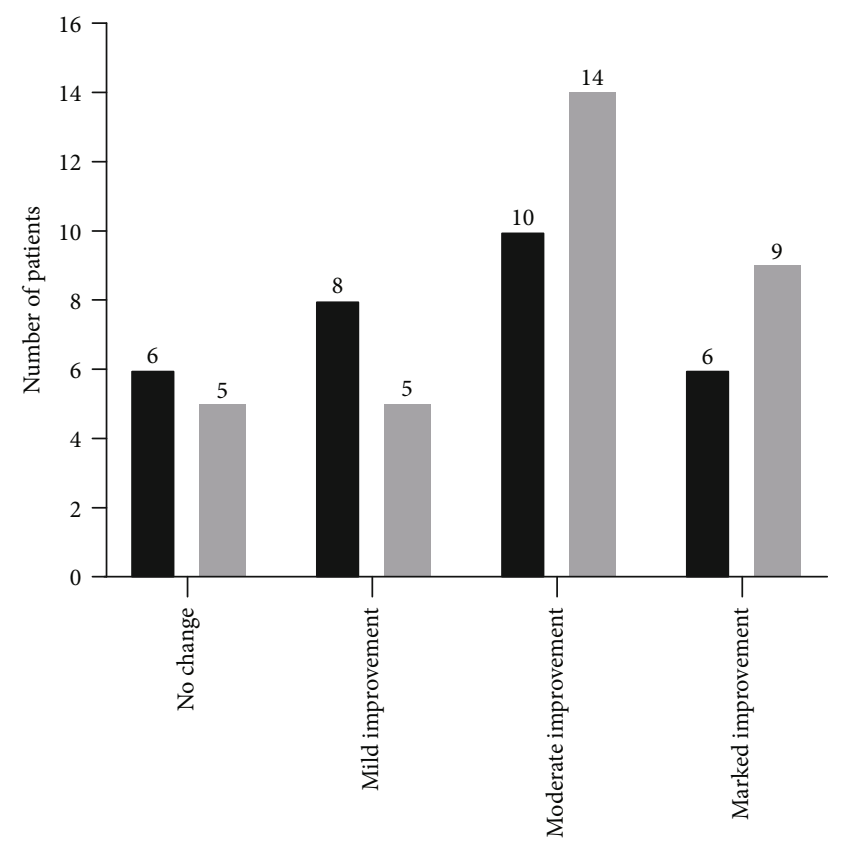

BTA BTA+FNS

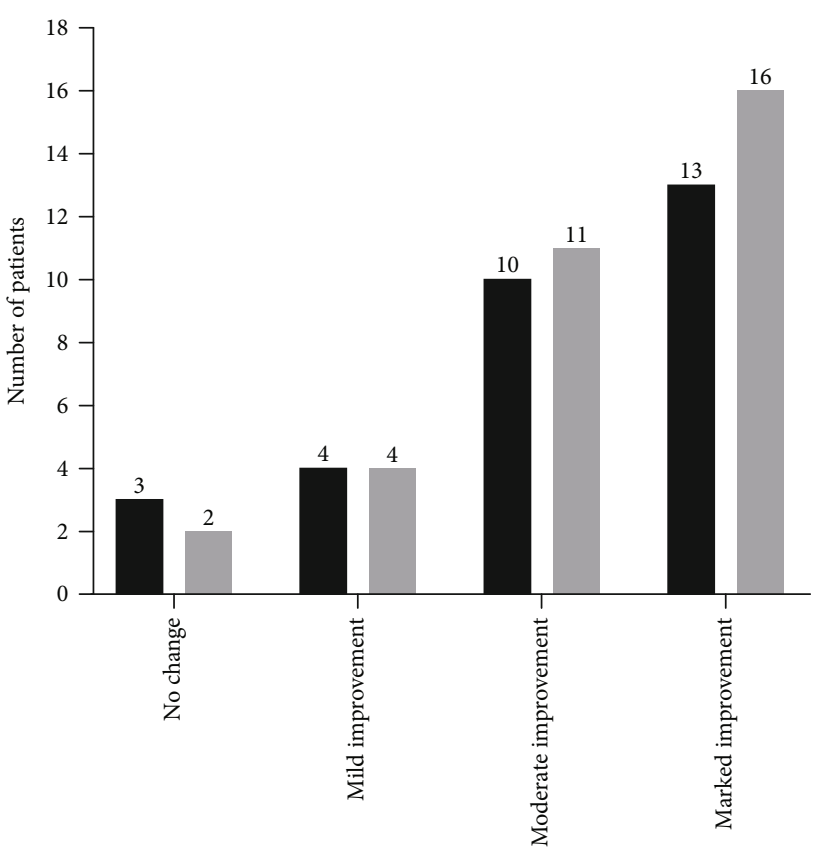

BTA
BTA+FNS

(a)

(b)

FIGURE 2: Self-assessment evaluation of patients based on questionnaires. (a) Degree of improvement on symptoms of scalp oil secretion, pruritus, and dandruff. (b) Degree of improvement on reduction in hair loss. BTA: botulinum toxin type A; BTA+FNS: botulinum toxin type A+finasteride.

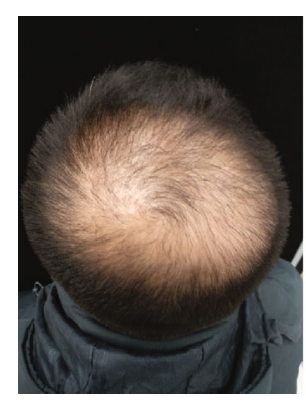

Before treatment

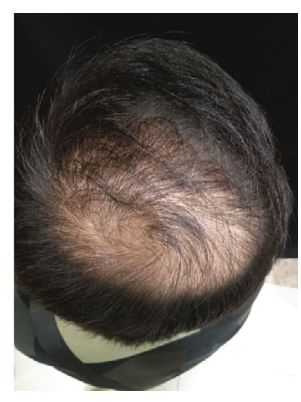

One month following the first treatment
Figure 3: Evaluation of clinical effects on growth and density of hairs one month following the first treatment with BTA.

data indicated that BTA was effective for the treatment of AGA without noticeable side effects and complications, and BTA combined with FNS exhibited a rapid and better therapeutic effect. Previous studies indicated that an increase in hair growth would only be observed with oral FNS for more than three months, and FNS's influence on hair density was poor on the frontal scalp and least effective bitemporally [28-30]. We found a significant improvement in hair growth and density in several patients one month after the first treatment (Figure 3). Also, compared with FNS treatment, we observed that BTA injection was more effective in regulating the hair density at the forehead and temples in some patients (Figure 4). However, these clinical characteristic findings
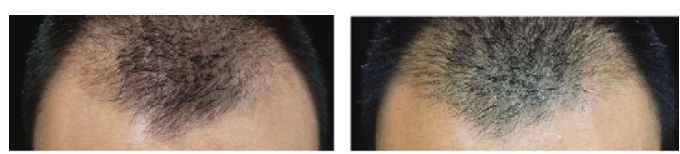

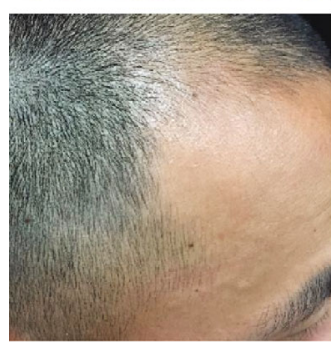

Before treatment

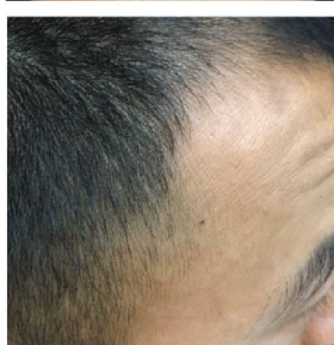

Once treatment
FIgURE 4: Evaluation of clinical effects on growth and density of hairs at forehead and temples following injection with BTA.

warrant further investigation. Moreover, the self-assessment of patients for scalp dandruff, pruritus and greasy scalp symptoms were significantly alleviated, and the thickness and texture of the hair were markedly ameliorated one month after the first treatment. We also found that treatment with BTA+FNS was less effective for AGA patients with diffuse hair loss (Figure 5(a)). In addition, we tried to use BTA for a female AGA patient, and our results indicated that BTA may increase growth and density of hairs as revealed by head photographs (Figure 5(b)). However, further clinical studies on a large sample size are needed to validate these findings. 

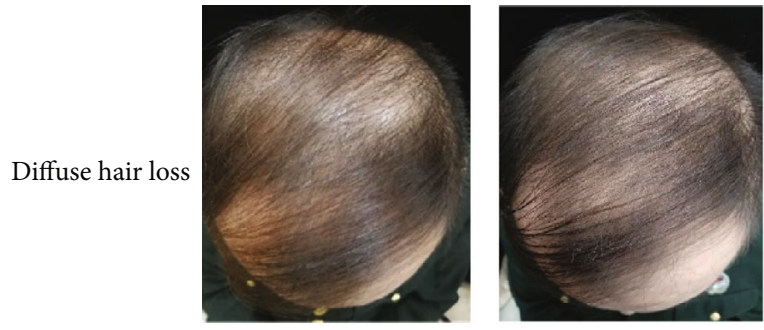

(a)

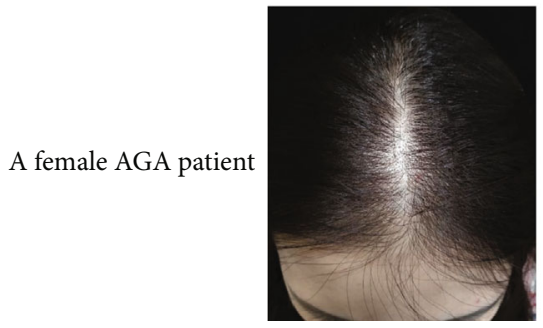

Before treatment

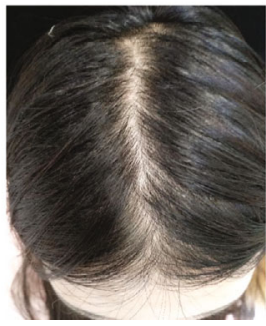

Once treatment

(b)
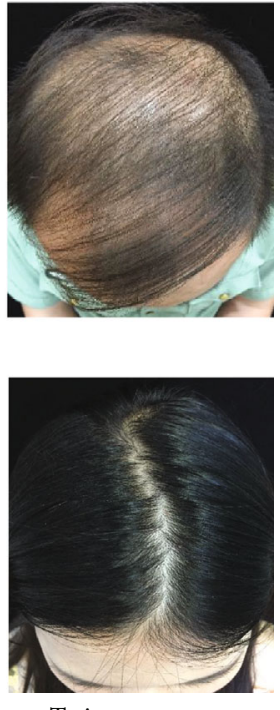

Twice treatment

FIgURE 5: Evaluation of clinical effects from (a) a AGA patient with diffuse hair loss and (b) a female AGA patient on growth and density of hairs following treatment with BTA+FNS.

This study still had several limitations. The number of study patients was only 63 , which means that additional studies including large populations are required. No evaluation of plasma and scalp DHT levels was assessed in our study. Excluding hair counts, other trichoscopic parameters, including dermoscopic images, hair diameter, and the proportion of miniaturized hairs could be used in monitoring treatment efficacy in the further study.

In conclusion, the findings of the present study demonstrated that BTA is a safe and effective therapy for the management of AGA and BTA combined with FNS presents excellent results. These data provide a novel theoretical basis and therapeutic strategy for the treatment of AGA.

\section{Data Availability}

The data used to support the findings of this study are included in this published paper and can be available from the corresponding author upon request.

\section{Disclosure}

The author is accountable for all aspects of the work in ensuring that questions related to the accuracy or integrity of any part of the work are appropriately investigated and resolved.

\section{Conflicts of Interest}

The authors declare that they have no conflicts of interest to disclose.

\section{Acknowledgments}

This work was supported by a grant from the Sanya Medical and Health Science and Technology Innovation Project (No. 2017YW15).

\section{References}

[1] Y. Kelly, A. Blanco, and A. Tosti, "Androgenetic alopecia: an update of treatment options," Drugs, vol. 76, no. 14, pp. 1349-1364, 2016.

[2] F. Lolli, F. Pallotti, A. Rossi et al., "Androgenetic alopecia: a review," Endocrine, vol. 57, no. 1, pp. 9-17, 2017.

[3] T. F. Cash, "The psychosocial consequences of androgenetic alopecia: a review of the research literature," The British Journal of Dermatology, vol. 141, no. 3, pp. 398-405, 1999.

[4] O. T. Norwood, "Incidence of female androgenetic alopecia (female pattern alopecia)," Dermatologic Surgery, vol. 27, no. 1, pp. 53-54, 2001.

[5] L. Yip, N. Rufaut, and R. Sinclair, "Role of genetics and sex steroid hormones in male androgenetic alopecia and female pattern hair loss: an update of what we now know," The Australasian Journal of Dermatology, vol. 52, no. 2, pp. 8188, 2011.

[6] R. M. Trueb, "Molecular mechanisms of androgenetic alopecia," Experimental Gerontology, vol. 37, no. 8-9, pp. 981-990, 2002.

[7] O. T. Norwood, "Male pattern baldness: classification and incidence," Southern Medical Journal, vol. 68, no. 11, pp. 13591365, 1975.

[8] G. D. Monheit and A. Pickett, “AbobotulinumtoxinA: a 25year history," Aesthetic Surgery Journal, vol. 37, Supplement 1, pp. S4-S11, 2017.

[9] O. H. Ababneh, A. Cetinkaya, and D. R. Kulwin, "Long-term efficacy and safety of botulinum toxin A injections to treat blepharospasm and hemifacial spasm," Clinical \& Experimental Ophthalmology, vol. 42, no. 3, pp. 254-261, 2014.

[10] D. Canfield, "Photographic documentation of hair growth in androgenetic alopecia," Dermatologic Clinics, vol. 14, no. 4, pp. 713-721, 1996.

[11] E. Ludwig, "Classification of the types of androgenetic alopecia (common baldness) occurring in the female sex," The British Journal of Dermatology, vol. 97, no. 3, pp. 247-254, 1977. 
[12] F. Xu, Y. Y. Sheng, Z. L. Mu et al., "Prevalence and types of androgenetic alopecia in Shanghai, China: a communitybased study," The British Journal of Dermatology, vol. 160, no. 3, pp. 629-632, 2009.

[13] T. L. Wang, C. Zhou, Y. W. Shen et al., "Prevalence of androgenetic alopecia in China: a community-based study in six cities," The British Journal of Dermatology, vol. 162, no. 4, pp. 843-847, 2010.

[14] D. R. Nyholt, N. A. Gillespie, A. C. Heath, and N. G. Martin, "Genetic basis of male pattern baldness," The Journal of Investigative Dermatology, vol. 121, no. 6, pp. 1561-1564, 2003.

[15] N. Orentreich, "Autografts in alopecias and other selected dermatological conditions," Annals of the New York Academy of Sciences, vol. 83, pp. 463-479, 1959.

[16] S. Inui and S. Itami, "Molecular basis of androgenetic alopecia: from androgen to paracrine mediators through dermal papilla," Journal of Dermatological Science, vol. 61, no. 1, pp. 1-6, 2011.

[17] S. W. Lee, M. Juhasz, P. Mobasher, C. Ekelem, and N. A. Mesinkovska, "A systematic review of topical finasteride in the treatment of androgenetic alopecia in men and women," Journal of Drugs in Dermatology, vol. 17, no. 4, pp. 457-463, 2018.

[18] F. Kaliyadan, A. Nambiar, and S. Vijayaraghavan, “Androgenetic alopecia: an update," Indian Journal of Dermatology, Venereology and Leprology, vol. 79, no. 5, pp. 613-625, 2013.

[19] I. G. Motofei, D. L. Rowland, D. L. Baconi et al., "Androgenetic alopecia; drug safety and therapeutic strategies," Expert Opinion on Drug Safety, vol. 17, no. 4, pp. 407-412, 2018.

[20] A. Rossi, C. Cantisani, M. Scarno, A. Trucchia, M. C. Fortuna, and S. Calvieri, "Finasteride, $1 \mathrm{mg}$ daily administration on male androgenetic alopecia in different age groups: 10-year follow-up," Dermatologic Therapy, vol. 24, no. 4, pp. 455461, 2011.

[21] A. Sato and A. Takeda, "Evaluation of efficacy and safety of finasteride $1 \mathrm{mg}$ in 3177 Japanese men with androgenetic alopecia," The Journal of Dermatology, vol. 39, no. 1, pp. 27-32, 2012.

[22] E. S. Felber, "Botulinum toxin in primary care medicine," The Journal of the American Osteopathic Association, vol. 106, no. 10, pp. 609-614, 2006.

[23] D. Monti, S. Tampucci, S. Burgalassi et al., "Topical formulations containing finasteride. Part I: _in vitro_permeation/penetration study and_in vivo_ pharmacokinetics in hairless rat," Journal of Pharmaceutical Sciences, vol. 103, no. 8, pp. 2307-2314, 2014.

[24] B. E. Goldman, D. M. Fisher, and S. L. Ringler, "Transcutaneous $\mathrm{PO}_{2}$ of the scalp in male pattern baldness: a new piece to the puzzle," Plastic and Reconstructive Surgery, vol. 97, no. 6, pp. 1109-1116, 1996.

[25] B. J. Freund and M. Schwartz, "Treatment of male pattern baldness with botulinum toxin: a pilot study," Plastic and Reconstructive Surgery, vol. 126, no. 5, pp. 246e-248e, 2010.

[26] S. Singh, S. Neema, and B. Vasudevan, "A pilot study to evaluate effectiveness of botulinum toxin in treatment of androgenetic alopecia in males," Journal of Cutaneous and Aesthetic Surgery, vol. 10, no. 3, pp. 163-167, 2017.

[27] L. Zhang, Q. Yu, Y. Wang, Y. Ma, Y. Shi, and X. Li, “A small dose of botulinum toxin $\mathrm{A}$ is effective for treating androgenetic alopecia in Chinese patients," Dermatologic Therapy, vol. 32, no. 4 , article e12785, 2019.
[28] V. Kanti, A. Messenger, G. Dobos et al., "Evidence-based (S3) guideline for the treatment of androgenetic alopecia in women and in men - short version," Journal of the European Academy of Dermatology and Venereology, vol. 32, no. 1, pp. 11-22, 2018.

[29] J. Leyden, F. Dunlap, B. Miller et al., "Finasteride in the treatment of men with frontal male pattern hair loss," Journal of the American Academy of Dermatology, vol. 40, no. 6, pp. 930937, 1999.

[30] K. York, N. Meah, B. Bhoyrul, and R. Sinclair, "A review of the treatment of male pattern hair loss," Expert Opinion on Pharmacotherapy, vol. 21, no. 5, pp. 603-612, 2020. 